\title{
A Neuro-Fuzzy Model for QoS Based Selection of Web Service
}

\author{
Abdallah Missaoui ${ }^{1}$, Kamel Barkaoui ${ }^{2}$ \\ ${ }^{1}$ LSTS-ENIT, Tunis, Tunisia; ${ }^{2}$ CEDRIC-CNAM, Paris, France. \\ Email: abdallah.missaoui@enit.rnu.tn, barkaoui@cnam.fr \\ Received January $5^{\text {th }}, 2010$; revised March $8^{\text {th }}, 2010$; accepted March $10^{\text {th }}, 2010$.
}

\begin{abstract}
The automatic selection and composition of Web services rely strongly on the manner to deal with ambiguity inherent to the description of functionalities of these services and the client's requests. Quality of Service (QoS) criteria become crucial in Web services selection and the problem of checking that a web service satisfies a given level of QOS is considered in recent research works. This paper presents a QoS based automatic classification method of web services. These services give generally similar functionalities and are offered by different providers. The main feature of our Web service selection model is to take advantage of the neuro-fuzzy logic for coping with the imprecision of QoS constraints values.
\end{abstract}

Keywords: Web Service, Selection, Neuro-Fuzzy, QoS, Constraint

\section{Introduction}

Web services are modular, self-contained, self-describing software components which are distributed over the Web. They can be readily located and checked-out online and dynamically, using a new directory and corresponding search mechanism known as Universal Description, Discovery, and Integration (UDDI).

The requester accesses the description using a UDDI or other types of registry, and requests the execution of the provider's service by sending a SOAP message to it (see Figure 1).

SOAP and HTTP provide exactly what they were designed for a simple, lightweight mechanism for interop-

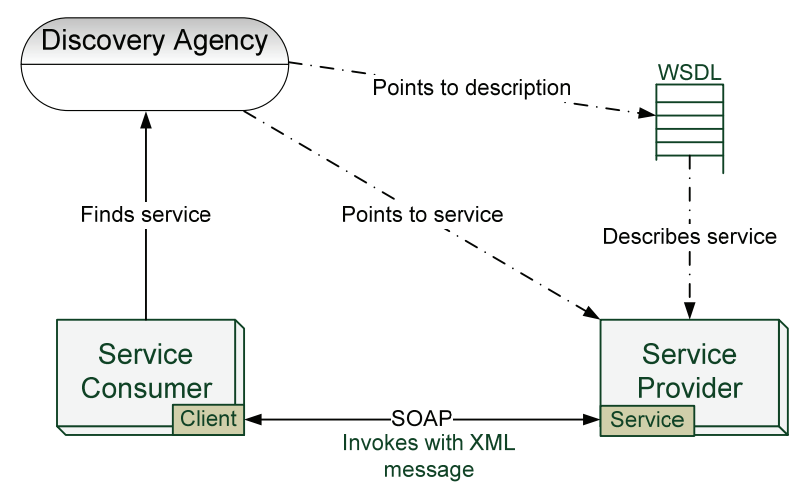

Figure 1. Basic web services architecture erability and distributed communication. However, SOAP and HTTP do not provide the traditional enterprise qualities of service typically needed for an enterprise.

Furthermore, SOAP was designed to be extensible, and it can be extended to support any desired QoS feature by adding SOAP headers to the SOAP messages and adding QoS features to the basic SOAP run-time facilities.

In recent years, several service providers offer QoS features to there customers. Then, multiple providers may provide similar functionalities with different values of non-functional properties.

Their non-functional properties need to be considered during service selection. There are characterised as quality of service (QoS). In many practice cases of business applications, it is recommended to be taken into account during the provider selection.

The human faculty of cognition and perception is very complex, but it possesses an efficient mechanism for information processing and expression $[1,2]$.

This paper applies the neuro-fuzzy decision making approach in the process of selection and choice of the most appropriate web service with respect to quality of service criteria.

This paper is organized as follows: Section 2 presents web service QoS generic description. In Section 3, we discuss and evaluate related works on web service selection adopting a common fuzzy logic approach. Section 4 , 
we enlighten our QoS requirement description model exploiting neuro-fuzzy logic in order to deal with the imprecision of QoS constraints values. Comments and recommendations for our model are explicitly presented in Section 5. Finally, Section 6 draws a conclusion.

\section{QoS Properties of Web Service}

Many services are appearing on the Web, several requesters are presented to a group of service providers offering similar services. Different service providers may have different qualities of service.

QoS is one of the most important factors for user's choice of Web service. This will require sophisticated patterns of selection process. It is necessary to provide an appropriate negotiation mechanism between clients and service providers to reach mutually-agreed QoS goals.

QoS management in Web service architecture includes the definition of QoS attributes and the specifications of the following processes: QoS publication, discovery, validation, and monitoring. Many works have studied QoS management on web service. Several QoS languages and architectures are proposed.

The proposed approaches for QoS management can be classified into two groups: one based on extending web service technologies including SOAP, WSDL and UDDI to support QoS [3-5]. The second group use independent entities to perform QoS management [6].

Quality of service is defined by the ability to provide different priorities to different applications, users, or data flow, or to guarantee a certain level of performance to a data flow. A QoS property may include several sub-properties representing different evaluation criteria, e.g. availability, performance, accessibility. In addition, a QoS property can be evaluated by many metrics and therefore it is necessary to define the units of measurements.

QoS in web service architecture is a combination of several qualities or properties of a service, such as:

- Response time: the interval between a usercommand and the reception of an action, a result or a feedback from the service.

- Availability: availability is the percentage of time that a service is available for use;

- Accessibility: Accessibility represents the degree that a system is normatively operated to counteract request messages without delay.

- Throughput: It means the max number of services that a platform providing Web services can process for a unit time.

- Reliability: Reliability is the quality aspect of a Web service that represents the degree of being capable of maintaining the service and service quality. The number of failures per month or year represents a measure of reliability of a Web service.

- Price: represents the money that the customer should pay for this service. It is always associated with the value of the service's functionality, i.e. the more a service costs, the more complicated functions it provides.

- Security Level: represents the security level of a service. It includes the existence and type of authentication mechanisms the service offers, confidentiality and data integrity of messages exchanged, non-repudiation of requests or messages, and resilience to denial-of-service attacks [7].

\section{Related Work}

With the strong popularity of the development of service oriented application, quality of service becomes a central interest of more and more researchers and enterprises. QoS values are proportional to the reliability degree and performance of service and thus play a very important role in the provider choice. A large number of services are exposed constraint information's for comparison providers.

Many researches [5,8-10] have studied QoS issues to improve two processes of discovery and selection of services. Several QoS-aware web service selection mechanisms have been developed in recent years in order to perform the web service composition and to improve performance applications based on services. This mechanisms' main objective is how to how properly select a set of providers that most satisfy the constraints defined by the user in his business processes.

Menascé studies the problem of finding services that minimize the total execution time. It presents an optimized heuristic algorithm that finds the optimal solution without exploring the entire solution space. The solution provided in [11] covers only the important case of execution constraints but not all QoS properties.

Pfeffer proposed a fuzzy logic based model for representing any kind of non-functional service properties. This representation of user preferences enables the fast evaluation of the requested service composition by a fuzzy multiplication of the service composition properties. Thus service composition' properties are measured during or after execution [12].

Other works have been done in fuzzy logic based web service selection. In [12-17], various methods have been proposed for specifying fuzzy QoS constraints and for ranking Web services based on their fuzzy representation.

There is a more suitable technique to quantify functional properties: Linear Programming. These properties are not fitting well for measuring the non-functional attributes, because the majority of them are not easy to be quantified in numerical form. In the meantime, user's QoS constraints regularly remain imprecise or ambiguous due to various human mental states, and it is very difficult to distinguish the priority order among QoS criteria.

Furthermore, in web services selection, the applied QoS constraints are not explicitly defined. It is necessary to relax the constraints to make an optimal solution. The use 
of fuzzy logic offers improvements in the overall satisfaction level. The QoS information's represented at abstract level such that it could efficiently select the best services.

However they are still initial efforts which need further investigation for more complete solutions. In the following, we specify several open issues that can be solved:

- When we use some kinds of fuzzy numbers like triangular fuzzy they may not be easy to be defined by end users.

- It is very important to correctly define the QoS properties that we use in the selection process. These criteria's QoS have important effects on ranking methods.

- How to improve fuzzy based web service discovery and the representation of QoS to achieve effective web service selection?

- How to automatically set the weights of service providers attributes?

\section{Refinement of the Framework}

Neuro-fuzzy technique is the combination of two artificial intelligence (AI) methods: fuzzy logic techniques and neural networks. Neuro-fuzzy system has the ability to handle the nonlinear and complex systems. It is constructed based on the learning algorithm of neural networks technique to adjust the appropriate parameters for fuzzy logic system [18].

In this paper, we aim to solve the selection of web services in a global and flexible manner by introducing a neuro-fuzzy way. For this purpose, we have developed a neural-fuzzy system based on the Sugeno Approach [19]. This is known as the ANFIS (i.e., Adaptive Neuro-Fuzzy Inference Systems). We assume that semantic matchmaking has taken place to identify functionally equivalent services. When several of them are available to perform the same task, their quality aspects become important and useful in the selection process.

An ANFIS is a multi-layered feed forward network, in which each layer performs a particular task. The layers are characterized by the fuzzy operations they perform. Figure 2 describes the global architecture of this neural-fuzzy system. It shows a $n$-input, type-5 ANFIS. Three membership functions are associated with each input.

We assume that the fuzzy inference system under consideration has $n$ inputs $Q_{1}, Q_{2}, \ldots, Q_{n}$ (which are one service attributes). Each input has five linguistic terms, for example, the input $Q_{1}$ possesses the terms $\left\{M_{11}, M_{12}, \ldots, M_{15}\right\}$.

For each input $Q_{i}$, we have defined linguistic expressions

$L_{i}=\{\operatorname{Very} \operatorname{Poor}(v p), \operatorname{Poor}(p), \operatorname{Medium}(m), \operatorname{Good}(g), \operatorname{Very}$ $\operatorname{Good}(v g)\}$

The common fuzzy if-then rule has the following type: Rule 1: If $\left(Q_{1}\right.$ is $\left.M_{11}\right)$ and $\left(Q_{2}\right.$ is $\left.M_{21}\right)$ and $\ldots$ and $\left(Q_{n}\right.$ is $\left.M_{n 1}\right)$ then $f_{1}\left(Q_{1}, Q_{2}, \ldots, Q_{n}\right)$
We denote the output of the $i^{\text {th }}$ node in layer $k$ as $O_{k, i}$. Figure 2 shows the schematic diagram of the ANFIS structure, which consists of five layers.

Layer 1: Every node $i$ in this layer transform the crisp values to a fuzzy one

$O_{1, i}=\mu_{M_{1 i}}\left(Q_{1}\right)$ for $i \in\{1,2, \ldots, 5\}$ and

$O_{1, i}=\mu_{M_{2 i}}\left(Q_{2}\right)$ for $i \in\{1,2, \ldots, 5\}$ and, $\ldots$, and

$O_{1, i}=\mu_{M_{n i}}\left(Q_{n}\right)$ for $i \in\{1,2, \ldots, 5\}$

where $Q_{K}$ is the input to node $K$ and $M_{k i}(k \in\{1, \ldots, n\}$ and $i \in\{1, \ldots, 5\}$ ) is a linguistic label (very poor, poor, fair, good, very good) associated with this node. In other words, $O_{I, i}$ is the membership grade of a fuzzy set $M \in\left\{M_{11}, \ldots, M_{15}\right\} \cup\left\{M_{21}, \ldots, M_{25}\right\} \cup \ldots \cup\left\{M_{n 1}, \ldots, M_{n 5}\right\}$ and it specifies the degree which the given input $Q_{K}$ $(k \in\{1, \ldots, n\})$ satisfies the quantifier $M$.

We use the following generalized Bell function as the membership function (MF)

$$
\mu_{M}(Q)=\frac{1}{1+\left|\frac{Q-c_{i}}{a_{i}}\right|^{2 b_{i}}}
$$

where $a_{i}, b_{i}$ and $c_{i}$ are the parameters set of MF. The bell-shaped function varies depending on the values of these parameters. Where the parameters $a$ and $b$ vary the width of the curve and the parameter $c$ locates the center

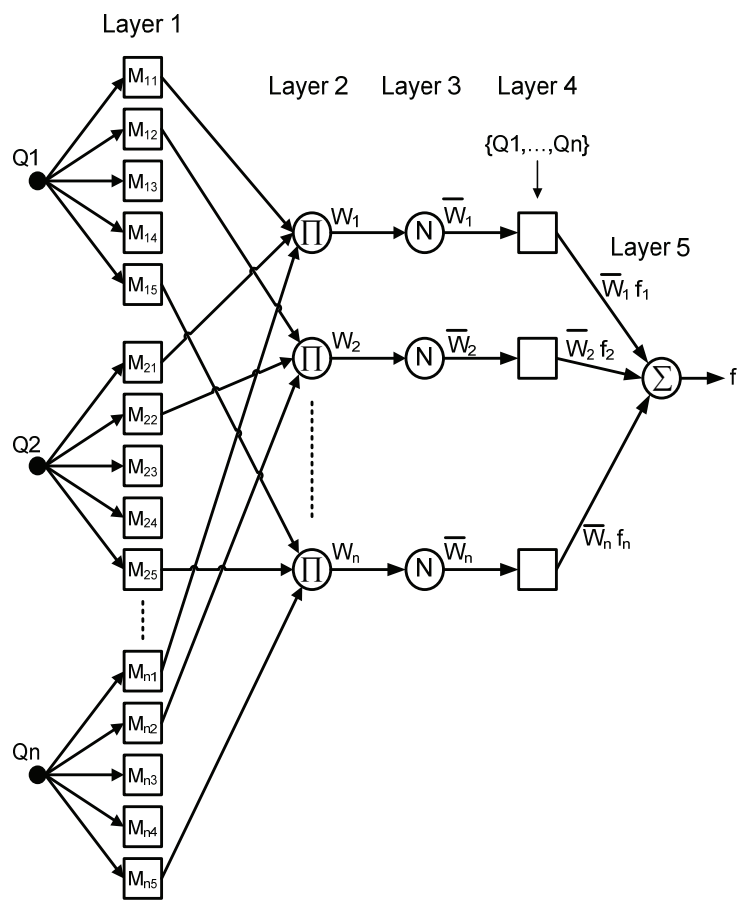

Figure 2. The structure of the neural fuzzy selector 
of the curve. The parameter $b$ should be positive. The parameters in this layer are referred to as premise parameters. The generalized Bell-shaped function is shown in Figure 3.

Layer 2: Every node in this layer is a fixed node labeled $\Pi$. The weighting factor, $w_{k}$, of each rule is calculated by evaluating the membership expressions in the antecedent of the rule. This is accomplished by first converting the input values to fuzzy membership values by utilizing the input membership functions and then applying the and operator to these membership values.

The and operator corresponds to the multiplication of input membership values.

$$
O_{2, i}=w_{i}=\mu_{M_{1 i}}\left(Q_{1}\right) \mu_{M_{2 i}}\left(Q_{2}\right) \ldots \mu_{M_{n i}}\left(Q_{n}\right)
$$
rule.

Each node output represents the firing strength of a

Layer 3: Every node in this layer is a fixed node labeled $\mathrm{N}$. The function of the fixed node is used to normalize the input firing strengths.

$$
O_{3, i}=\overline{w_{i}}=\frac{w_{i}}{\sum_{j=1}^{n} w_{j}} \quad i \in\{1, \ldots, n\}
$$

Layer 4: Every node in Layer 4 is a parameterized function, and the adaptive parameters are called "consequent parameters".

The node function is given by:

$$
O_{4, i}=\overline{w_{i}} f_{i}=\overline{w_{i}}\left(p_{1}^{i} Q_{1}+p_{2}^{i} Q_{1}+\ldots+p_{n}^{i} Q_{1}+p_{n+1}^{i}\right)
$$

Layer 5: The single node in this layer is a fixed node labeled $\sum$, which computes the overall output as the summation of all inputs:

$$
O_{5,1}=\sum_{i=1}^{n} \overline{w_{i}} f=\frac{\sum_{i=1}^{n} w_{i} f}{\sum_{i=1}^{n} w_{i}}
$$

Thus, the ANFIS network is constructed according to the TSK fuzzy model. This ANFIS architecture can then update its parameters according to the backpropagation algorithm [20].

This algorithm minimizes the error between the output

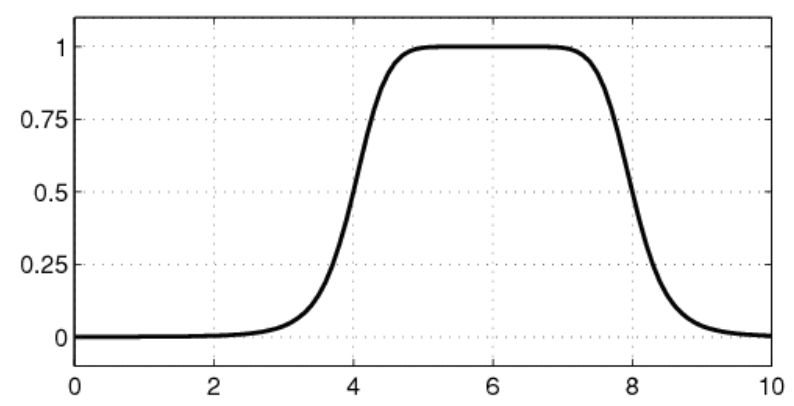

Figure 3. Generalized bell-shaped $(a=2, b=4, c=6)$ of the ANFIS and the desired output.

Our neuro-fuzzy system allows classifying service providers in several categories: very poor, poor, fair, good, very good. It allows automating the selection process in the dynamic composition of services.

According to the QoS requirements of web service providers and the functions of Neuro-fuzzy system, we believe that each service invoked is appropriate candidate to increase the composition ability of web services and to decrease the burden of composition cognition and the minimal development cost.

\section{Comments and Recommendations}

In fuzzy inference system (FIS), The MF of the consequent of each rule is a constant of a fuzzy MF. There are two steps to construct this system: the specification of an appropriate number of input/output and the specification of the shape of MFs. The main problem is that structure identification requires human expertise to solve the parameter estimation. In our selector system we used a different approach, which take advantage of adaptive neural networks algorithms during fitting procedures. MF parameters are fitted to a dataset through a learning algorithm.

A significant number of samples of service providers are needed in order to have better result and to avoid having too many defect values during selection process. The database must be as complete as possible, including samples of providers attributes over a broad range. The number of samples depends on the context and on the runtime environment.

On the other hand, fuzzy logic sets are based on transparence, linguistic rules and establish a framework to include human expertise into modelling. The number of rules is decided by an expert who is familiar with the system to be modeled. In our work, however, no expert is available and the number of membership functions assigned to each input qualities is chosen empirically by examining the desired input-output data.

We merged the fuzzy logic approach with the ability of learning algorithms from neural networks to adjust the model.

\section{Conclusions}

Web service composition is an emerging area involving important technological challenges. Some of the main challenges are to correctly describe QoS of Web services, to compose them adequately and automatically, and to discover suitable providers and QoS composition issues.

Neuro-fuzzy logic can be seen as a promising formal technique for representing imprecise QoS constraints. In this paper, we have presented a solution to use neurofuzzy approach in Web service discovery and selection. We have proposed methods for ranking and selecting web services based on a neuro-fuzzy specification of fuzzy 
QoS constraints. The user's constraints are formalized as fuzzy sets and the Qos criteria's are expressed as fuzzy expressions.

This model can be seen as a contribution towards a more complete solution for web service composition integrating fully QoS features.

\section{REFERENCES}

[1] J. S. Jang, "ANFIS: Adaptive-Network-Based Fuzzy Inference System," IEEE Transactions on Systems, Man, and Cybernetics, Vol. 23, No. 3, 1993, pp. 665-684.

[2] J. R. Jang and C. T. Sun, "Neuro-Fuzzy and Soft Computing: A Computational Approach to Learning and Machine Intelligence,” Prentice-Hall, Inc., Upper Saddle River, New Jersy, 1997.

[3] V. Diamadopoulou, C. Makris, Y. Panagis and E. Sakkopoulos, "Techniques to Support Web Service Selection and Consumption with QoS Characteristics," Journal of Network and Computer Applications, Vol. 31, No. 2, 2008, pp. 108-130.

[4] A. F. M. Huang, C. W. Lan and S. J. H. Yang, "An Optimal QoS-Based Web Service Selection Scheme," Information Sciences, Vol. 179, No. 19, 2009, pp. 3309-3322.

[5] L. Zeng, B. Benatallah, A. H. H. Ngu, M. Dumas, J. Kalagnanam and H. Chang, "QoS-Aware Middleware for Web Services Composition," IEEE Transactions on Software Engineering, 2004, pp. 311-327.

[6] D. A. Menascé, H. Ruan and H. Gomaa, "QoS Management in Service-Oriented Architectures," Journal of Performance Evaluation, Vol. 64, No. 7-8, 2007, pp. 646-663.

[7] D. A. Menasce, "QoS Issues in Web Services," IEEE Internet Computing, Vol. 6, No. 6, 2002, pp. 72-75.

[8] M. Sultana, M. M. Akbar and M. Rouf, "Network Flow Heuristic Algorithm for a Distributed Web Service Selection Problem," IEEE Conference on Communications, Computers and Signal Processing, 2009, pp. 465-470.

[9] D. Tsesmetzis, I. Roussaki and E. Sykas, "QoS-Aware Service Evaluation and Selection," European Journal of Operational Research, Vol. 191, No. 3, 2008, pp. 11011112.

[10] S. Chaari, Y. Badr and F. Biennier, "Enhancing Web Service Selection by QOS-Based Ontology and WS-Policy,"
Proceeding of the 23rd ACM Symposium on Applied Computing, Ceará, 2008, pp. 2426-2431.

[11] D. A. Menascé, E. Casalicchio and V. Dubey, "On Optimal Service Selection in Service Oriented Architectures," Performance Evaluation Journal, Vol. 67, No. 8, 2010, pp. 659-675.

[12] H. Pfeffer, S. Krüssel and S. Steglich, "A Fuzzy Logic based Model for Representing and Evaluating Service Composition Properties," The Third International Conference on Systems and Networks Communications, Bangalore, 2009.

[13] M. Lin, J. Xie, H. Guo and H. Wang, "Solving Qos-Driven Web Service Dynamic Composition as Fuzzy Constraint Satisfaction," IEEE International Conference on e-Technology, e-Commerce and e-Service, Hong Kong, 2005.

[14] P. Wang, K. Chao, C. Lo, C. Huang and Y. Li, "A Fuzzy Model for Selection of QoS-Aware Web Services," IEEE International Conference on e-Business Engineering, IEEE Computer Society, Shanghai, 2006, pp. 585-593.

[15] K. M. Chao, M. Younas, C. C. Lo and T. H. Tan, "Fuzzy Atchmaking for Web Services," The 19th International Conference on Advanced Information Networking and Applications, Taipei, 2005.

[16] L. Zhuang, Y. F. Huang, W. G. Jian, J. B. Zhou and H. Q. Guo, "Solving Fuzzy QoS Constraint Satisfaction Technique for Web Service Selection," International Conference on Computational Intelligence and Security Workshops, Harbin, 2007.

[17] H. Tong and S. Zhang, "A Fuzzy Multi-Attribute Decision Making Algorithm for Web Services Selection Based on QoS," The IEEE Asia-Pacific Conference on Services Computing, Guangzhou, 2006.

[18] M. A. Denai, F. Palis and A. Zeghbib, "ANFIS Based Modelling and Control of Non-Linear Systems: A Tutorial," IEEE International Conference on Systems, Man and Cybernetics, Vol. 4, 2004, pp. 3433-3438.

[19] O. Nelles, A. Fink, R. Babuka and M. Setnes, "Comparison of Two Construction Algorithms for TakagiSugeno Fuzzy Models," International Journal of Applied Mathematics and Computer Science, 2000, pp. 835-855.

[20] P. Werbos, "The Toots of the Back Propagation: From Ordered Derivatives to Neural Networks and Political Forecasting," John Wiley and Sons, Inc, New York, 1993. 